\title{
Progress in Percutaneous Penetration Enhancers from Traditional Chinese Medicines
}

\author{
Yingying Huang, ${ }^{a}$ Jinyu $\mathrm{Yi}^{,}{ }^{a}$ and Chaoyan Zhang ${ }^{*, a, b, c, d}$ \\ ${ }^{a}$ College of Food Science and Technology, Shanghai Ocean University, Shanghai 201306, China \\ ${ }^{b}$ Shanghai Engineering Research Center of Aquatic-Product Processing \& Preservation, \\ Shanghai 201306, China \\ ${ }^{c}$ Laboratory of Quality and Safety Risk Assessment for Aquatic Product on Storage and Preservation \\ (Shanghai), Ministry of Agriculture, Shanghai 201306, China \\ ${ }^{d}$ Collaborative Innovation Center of Seafood Deep Processing, Dalian Polytechnic University, \\ Dalian, Liaoning 116034, China
}

Email: chyzhang@shou.edu.cn (C. Z.)

\begin{abstract}
Percutaneous penetration enhancers can regulate the transdermal absorption of drugs. Generally speaking, it can be divided into two categories: chemical synthesis and natural sources. Natural percutaneous penetration enhancers have the advantages of safety and mildness. This review summarizes the mechanism and characteristics of three kinds of percutaneous penetration enhancers and their combined application in transdermal drug delivery systems. The three different types of percutaneous penetration enhancers are fatty acids, alkaloids and terpenes. It is expected to provide considerations for the application research of percutaneous penetration enhancers rich in traditional Chinese medicines in transdermal drug delivery systems.
\end{abstract}

Keywords traditional Chinese medicines, percutaneous penetration enhancers, combined application

\section{Introduction}

Transdermal drug delivery systems (TDDS) generally refer to a new type of drug delivery route, through which drugs are absorbed into the human blood circulation through the skin and reach an effective blood concentration. ${ }^{[1]}$ The drugs that enter the human blood circulation through this way can not only avoid the first pass effect of the liver, but also can effectively avoid the influence of factors such as digestive juice and gastrointestinal enzymes in the gastrointestinal tract, thereby improving the bioavailability. ${ }^{[2]}$ Patients can not only reduce the number of medications and improve the efficacy, but also achieve self-medication by TDDS. It is this unique advantage of transdermal drug delivery that has attracted attention. ${ }^{[3]}$ The focus in TDDS is mainly on how to improve the bioavailability of transdermal drug delivery. Percutaneous penetration enhancers can regulate the transdermal absorption of drugs. At present, common percutaneous penetration enhancers include chemical percutaneous penetration enhancers and natural percutaneous penetration enhancers. ${ }^{[4]}$ Although chemical percutaneous penetration enhancers can greatly increase the transdermal permeability of drugs, it has been reported that a small amount of chemical percutaneous penetration enhancers can cause inflammation and skin irritation during transdermal administration. This problem greatly limits the clinical application of percutaneous penetration enhancers in transdermal administration. ${ }^{[5]}$ In this review, the percutaneous penetration enhancers from traditional Chinese medicines are divided into three categories, namely fatty acids, alkaloids and terpenes. A large number of studies have disclosed that percutaneous penetration enhancers containing traditional Chinese medicine active ingredients not only have their own pharmacological effects, but also have little side effects on the body and skin. ${ }^{[6]}$ If these advantages and the sustainability of natural resources can be fully utilized, the percutaneous penetration enhancers from traditional Chinese medicines will be more and more popular in the market.

\section{Main Barrier}

Transdermal administration can be passed through the epidermal route and the skin appendage route, which are the two major routes for transdermal absorption of drugs. Studies have shown that skin appendages account for only $1 \%$ of adult skin surface area of $1.7 \mathrm{~m}^{2}$, although the drug passing rate of skin appendages is high. ${ }^{[7]}$ Therefore, the complete epidermal absorption route is the main route of drug absorption. The epidermal absorption pathway refers to the way that drugs diffuse through the epithelial epidermis to the dermis to be absorbed by capillaries, eventually enter the human circulation. As shown in Figure $1 \mathrm{~A}$, the epithelial epidermal layer is composed of an active epidermal layer and a stratum corneum. ${ }^{[8]}$ The former is an aqueous tissue without blood vessels, and the transfer of metabolites and nutrients is mainly achieved by diffusion, which can't effectively prevent the invasion of foreign objects. The latter is a "brick wall structure" composed of keratinocytes and lipid bilayers. This structure makes the stratum corneum very tough and is the main barrier for transdermal absorption of drugs. ${ }^{[9]}$ Guo et al. ${ }^{[10]}$ divided the epidermal absorption pathway into intercellular pathway and transcellular pathway according to the different ways of drug passing through the stratum corneum. As shown in Figure 1B, it can be seen that the drugs in the transcellular pathway bypass the keratinocytes to reach the active epidermis. The length of the pathway is significantly longer than that of the intercellular pathway, but the permeability of the keratinocytes is low and the intercellular pathway needs to repeat the distribution process of the hydrophilic/lipophilic environment.

Therefore, the transcellular pathway accounts for a large proportion in the epidermal absorption pathway. After confirm- 


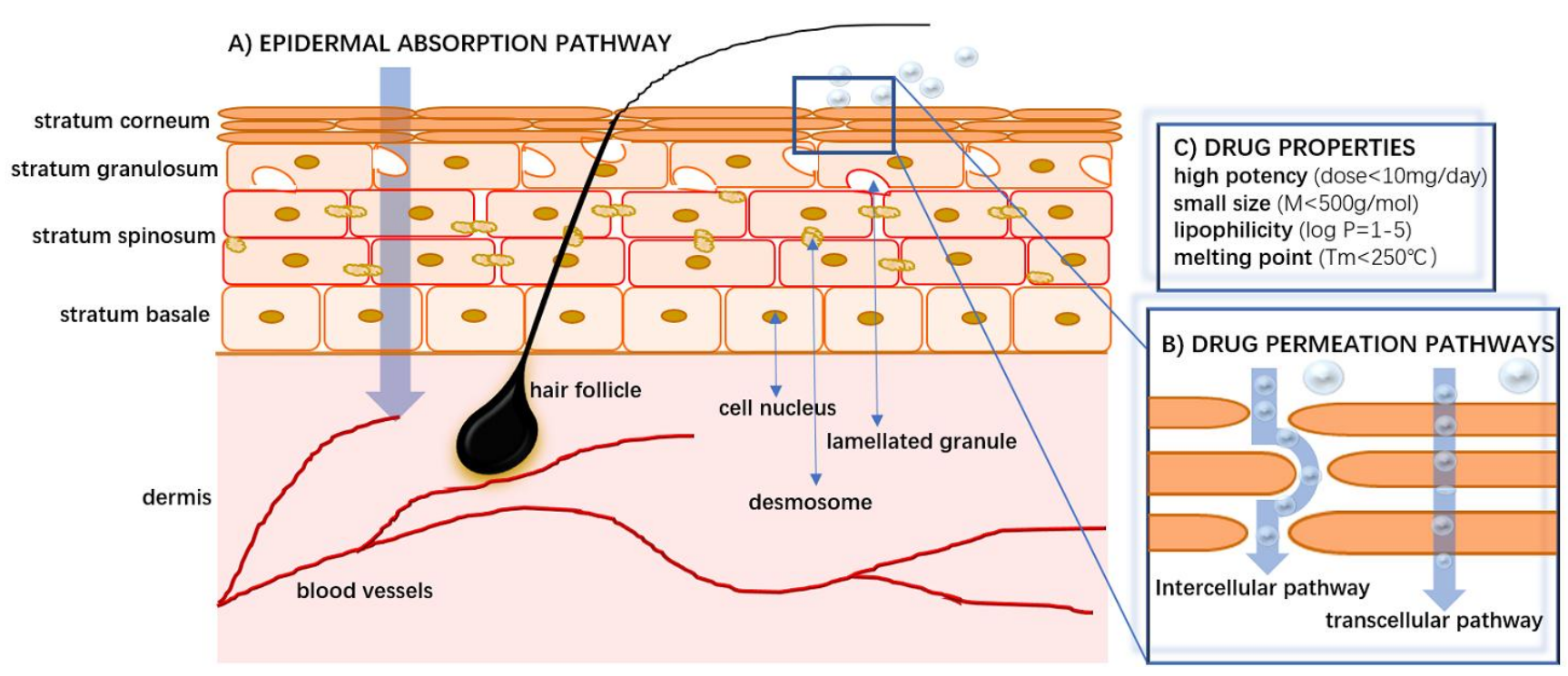

Figure 1 The structure of skin layer.

Table 1 Traditional Chinese medicines with percutaneous penetration promoting effect

\begin{tabular}{ccc}
\hline Traditional Chinese medicine name & Main percutaneous penetration enhancer & Ingredient type \\
\hline Myristica fragrans & Myristic acid ${ }^{[14]}$ & Fatty acids \\
Rapeseed & Oleic acid ${ }^{[15,16]}$ & \\
\hline Rhizoma Chuanxiong & Tetramethylpyrazine $^{[17]}$ & Alkaloids \\
Coptis chinensis & Berberine $^{[18]}$ & \\
Evodia rutaecarpa & Evodiamine $^{[19,20]}$ & Terpenoids \\
Sophora flavescens & Matrine $^{[21,22]}$ & \\
\hline Eucalyptus leaves & Cineole $^{[23]}$ & \\
Borneolum synthcticum & Borneol $^{[24]}$ & Menthol $^{[25]}$ \\
Mentha piperita & &
\end{tabular}

ing that the lipid bilayer in the stratum corneum is the main factor causing the change of skin resistance value, Zhu et al. ${ }^{[11]}$ used the change of skin resistance value as a rapid screening criterion for percutaneous penetration enhancers, further explained that the stratum corneum is the main barrier of drug transdermal absorption, especially the lipid bilayer in the stratum corneum.

\section{Percutaneous Penetration Enhancers}

The main barrier of TDDS is the stratum corneum, which is highly selective for the types of molecules allowed to pass through the skin, ${ }^{[12,13]}$ as shown in Figure $1 \mathrm{C}$. Therefore, percutaneous penetration enhancers need to be added to the preparation to improve the bioavailability of transdermal drug delivery. The main natural substances used in percutaneous penetration enhancers are traditional Chinese medicines or its active ingredients. The commonly used traditional Chinese medicines include Myristica fragrans, Rhizoma Chuanxiong, Sophora flavescens, Borneolum synthcticum, Mentha piperita, and so on (Table 1).

The components of traditional Chinese medicines are complex and diverse, and the monomer components can't fully represent the effective components of percutaneous penetration promotion. In addition to the three component types discussed in this review, there are other compounds that also have percutaneous penetration promotion. However, fatty acids, ${ }^{[26]}$ alkaloids ${ }^{[27]}$ and terpenes ${ }^{[28]}$ are the most studied compounds in the field of percutaneous penetration promotion in recent years, and they are also easily obtained from natural sources.

\section{Fatty acids}

In recent years, there is careful research on natural percutaneous penetration enhancers, especially those containing fatty acids. Fatty acids are composed of aliphatic hydrocarbon chains and terminal carboxyl groups. In general, most fatty acids are straight chain compounds with carbon chain length between 2 and 24. ${ }^{[29]}$ Medium and long-chain fatty acids are often used as percutaneous penetration enhancers (Figure 2). ${ }^{[30]}$ It has been reported that the fatty acids in medicinal plants can be divided into three types according to the carbon atom structure: saturated fatty acids, monounsaturated fatty acids and polyunsaturated fatty acids. ${ }^{[31]}$ Among them, saturated fatty acids (lauric acid ${ }^{[32]}$ ) and unsaturated fatty acids (oleic acid ${ }^{[33,34]}$ ) have good percutaneous penetration ability.

Jain et al. ${ }^{[35]}$ indicated that fatty acids can increase the percutaneous permeability of tricyclic antidepressants because of the increased fluidity of lipid bilayers between keratinocytes. Blake et $a l^{[36]}$ found that fatty acids increase the fluidity of lipid 
<smiles>CCCCCCCCCCCC(=O)O</smiles>

Lauric acid

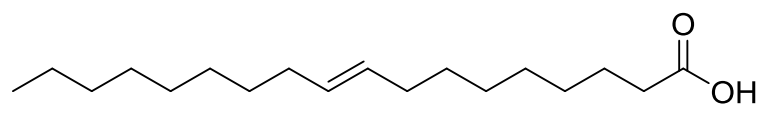

Oleic acid

Figure 2 The structures of fatty acids with different saturations.

bilayers by disrupting the arrangement of lipid alkyl chains in the stratum corneum. Aungst et al ${ }^{[37]}$ found that unsaturated fatty acids were better than corresponding saturated isomers as percutaneous penetration enhancers. Xi et al. ${ }^{[38]}$ indicated that the mechanism of promoting percutaneous penetration of oleic acid is caused by its chemical structure. Oleic acid is an unsaturated fatty acid containing an unsaturated double bond (Figure 2). At this time, the existence of the unsaturated double bond makes its spatial structure asymmetric, increasing the fluidity of the lipid bilayer in the stratum corneum, thereby increasing the percutaneous permeability of transdermal administration. Therefore, unsaturated fatty acids have better percutaneous promotion than saturated fatty acids.

\section{Alkaloids}

Alkaloids are a class of nitrogen containing organic compounds, which are generally alkaline and can form salts with various acids, ${ }^{[39]}$ such as Scopolamine (Figure 3). Scopolamine is the first alkaloid drug produced commercially in transdermal administration. ${ }^{[40]}$ Due to the pharmacological properties of alkaloids, alkaloids as percutaneous penetration enhancers have been a research hotspot. After comparing the results of percutaneous absorption of various alkaloids, Wang ${ }^{[41]}$ found that the percutaneous penetration enhancers containing alkaloids had good transdermal effect. Li et al. ${ }^{[42]}$ verified evodiamine, sinapine thiocyanate and berberine hydrochlori can promote the transdermal absorption of paeoniflorin in vitro.<smiles>CN1C2COC(OC(=O)C(CO)c3ccccc3)CC1C2</smiles>

Figure 3 The structure of scopolamine.

$\mathrm{Xu}^{[43]}$ found a certain relationship between the absorption coefficient of alkaloids and the oil-water partition coefficient of drugs in vitro. When alkaloids are used as percutaneous penetration enhancers, the oil-water distribution and molecular weight of the drugs should be given priority. $\mathrm{Li}$ et al. ${ }^{[44]}$ compared the stratum corneum of normal mouse skin and the stratum corneum treated by matrine by means of electron microscopy and staining, and found that Sophora flavescens as a natural percutaneous penetration enhancer can promote the percutaneous penetration by damaging the stratum corneum of mouse skin.

\section{Terpenes}

Terpenoids are a kind of compounds derived from methyl glutaric acid and the molecular formula conforms to the general formula $\left(\mathrm{C}_{5} \mathrm{H}_{8}\right)_{n}$. They can be classified according to the number of isoprene units in the molecular structure, such as monoterpenes, sesquiterpenes, diterpenes, etc. Furthermore, the US Food and Drug Administration listed terpenoids as safe and effective percutaneous penetration enhancers. ${ }^{[45}$ Terpenoids can not only enhance the percutaneous permeability of lipophilic drugs, but also enhance the percutaneous permeability of hydrophilic drugs. ${ }^{[46]}$ Strong lipophilicity is an important structural feature of terpene compounds as lipophilic drug accelerators (Figure 4). ${ }^{[47]}$ Vaddi et al. ${ }^{[48]}$ found that terpenes, such as carvacrol, linalool and a-terpineol, could increase the percutaneous permeability of haloperidol. Studies have shown that smaller terpenes with higher unsaturation and terpenes with very low unsaturation can enhance the percutaneous permeability of hydrophilic drugs (Figure 5). ${ }^{[49]}$ Shen et al. ${ }^{[50]}$ found that the terpenoid carvone, 1,8-cineole and thymol can be used as percutaneous penetration enhancers of the hydrophilic drug 5 -fluorouracil.<smiles>Cc1ccc(C(C)C)cc1O</smiles>

Carvacro<smiles>CC1=CCC(C(C)(O)O)CC1</smiles>

a-Terpineol<smiles>C=CC(C)(O)CCC=C(C)C</smiles>

Linalool
Figure 4 The structures of terpenes in the percutanous absorption of haloperidol.<smiles>C=C(C)C1CC=C(C)C(=O)C1</smiles>

Carvone<smiles>CC12CCC(C1)C(C)(C)O2</smiles>

1,8-Cineole<smiles>Cc1ccc(C(C)C)c(O)c1</smiles>

Thymol
Figure 5 The structures of terpenes in the percutanous absorption of 5 -fluorouracil.

A large number of ceramides are closely arranged in the lipid bilayers of the stratum corneum through hydrogen bond. It is hydrogen bond that forms a network at the head of ceramide, which makes the lipid bilayer structure firm and stable. Tight networks may be relaxed by terpenes with functional groups that provide or accept a hydrogen bond, such as oxygenated monoterpenes. ${ }^{[51]}$ Wang et al. ${ }^{[52]}$ found that the functional groups of terpenoids preferentially combined with ceramide in the stratum corneum to form hydrogen bonds by Fourier transform infrared spectroscopy (FTIR), thus improving the drug percutaneous permeability. Some scholars believe that terpenoids can also play a role in the intercellular pathway of drugs. Rutile et al. ${ }^{[53]}$ observed that different concentrations of terpene can change the configuration of keratin in the stratum corneum by FTIR. Therefore, the mechanism of terpenoids may promote the change of the configuration of keratin in the stratum corneum to promote the transdermal absorption of drugs.

\section{Percutaneous Penetration Enhancers in Chinese Medicine Prescriptions}

The single percutaneous penetration enhancer from traditional Chinese medicines suffers from some limitations. Thus, we can try to combine multi-component Chinese medicine components to increase its percutaneous penetration. ${ }^{[54,55]}$ Berberine as alkaloids can increase the range 
of drugs for transdermal administration. Liu et al. ${ }^{[56]}$ found that berberine, as a percutaneous penetration enhancer in Jinhuang salve, had a slow penetration rate and a long lag time. However, other components of the compound, such as Pedate pinallia jackinthepulpit rhizome, Pericarpium citri reticulatae viride and Magnolia officinalis, etc., could promote the percutaneous permeation. Yang et al. ${ }^{[57]}$ found that Borneolum synthcticum is a natural percutaneous penetration enhancer, which not only has its own pharmacological effect, but also can promote the transdermal absorption of matrine.

\section{Conclusions}

In this review, the percutaneous penetration enhancers from traditional Chinese medicines are divided into three categories: fatty acids, alkaloids and terpenes. It can be seen from the review that percutaneous penetration enhancers from traditional Chinese medicines not only have pharmacological effects, but also have little side effects on the body and skin. The bioavailability of transdermal delivery can be improved by adding percutaneous penetration enhancers to the prescription of traditional Chinese medicines. The combination of different components of traditional Chinese medicines can also improve its percutaneous permeability. In recent years, researchers have paid more attention to effectively improving the percutaneous penetration of drugs. For example, percutaneous penetration enhancers from traditional Chinese medicines can also be combined with physical technologies such as ion percutaneous penetration, ${ }^{[58]}$ electroporation, ${ }^{[59]}$ microneedles, ${ }^{[60]}$ etc.

\section{Conflict of Interest}

The authors declare no conflict of interest.

Copyright (C) 2020 Yingying Huang, Jinyu Yi, Chaoyan Zhang. This article is an open access article distributed under the terms and conditions of the Creative Commons Attribution (CC BY) license (http://creativecommons.org/licenses/by/4.0/). The use, distribution or reproduction in other forums is permitted, provided the original author(s) or licensor are credited and that the original publication in this journal is cited, in accordance with accepted academic practice. No use, distribution or reproduction is permitted which does not comply with these terms.

\section{References}

[1] Kumar, S. V.; Tarun, P.; Kumar, T. A. Transdermal drug delivery system for non-steroidal anti-inflammatory drugs: a review. Indo Am. J. Pharm. Res. 2013, 5, 3585-3605.

[2] Bariya, S. H.; Gohel, M. C.; Mehta, T. A. Microneedles: an emerging transdermal drug delivery system. J. Pharm. Pharmacol. 2012, 64, 11-29.

[3] Chen, Y.; Quan, P.; Liu, X. C. Novel chemical permeation enhancers for transdermal drug delivery. Asian J. Pharm. Sci. 2014, 9, 51-64.

[4] Patil, U. K.; Saraogi, R. Natural products as potential drug permeation enhancer in transdermal drug delivery system. Arch. Dermatol. Res. 2014, 306, 419-426.

[5] Anubhav Arora, E. K.; Pankaj, K. Multicomponent chemical enhancer formulations for transdermal drug delivery: more is not always better. J. Control. Release 2010, 144, 175-180.

[6] Lan, Y. Li.; Hui, C. Y. Essential oil from zanthoxylum bungeanummaxim and its main components used as transdermal penetration enhancers: a comparative study. J. Zhejiang Univ. Sci. B 2014, 15, 940-952.

[7] Trommer, H.; Neubert, R. H. Overcoming the stratum corneum: the modulation of skin penetration. Skin Pharmacol. Physiol. 2006, 19,
$106-121$.

[8] Madison, K. C. Barrier function of the skin: "La Raison d'Être" of the epidermis. J. Invest. Dermatol. 2003, 121, 231-241.

[9] Menon, G. K.; Cleary, G. W.; Lane, M. E. The structure and function of the stratum corneum. Int. J. Pharm. 2012, 435, 3-9.

[10] Guo, P.; Li, A.; Long, J. An evaluation of mathematical models for predicting skin permeability. J. Pharm. Sci. 2008, 97, 584-598.

[11] Zhu, X. M.; Li, Y.; Xu, F. Skin electrical resistance measurement of oxygen containing terpenes as penetration enhancers: role of stratum corneum lipids. Molecules 2019, 24, 523.

[12] Andrej, K.; Monika, K.; Katerina, V. Permeation enhancers in transdermal drug delivery: benefits and limitations. Expert Opin. Drug Deliv. 2020, 17, 145-155.

[13] Naik, A.; Kalia, Y. N.; Guy, R. H. Transdermal drug delivery: overcoming the skin's barrier function. Pharm. Sci. Technol. Today 2000, 3, 318-326.

[14] Guo, P.; Li, D. Comparative study on fatty acid composition of nutmeg and nutmeg. Shihezi Sci. Technol. 2013, 4, 16-18 (in Chinese).

[15] Hao, Y.; Wu, W. Near infrared spectroscopy analysis model of oleic acid and linoleic acid in camellia oil. Acta Opt. Sin. 2019, 39, 381-386 (in Chinese).

[16] Niu, L. Y.; Yang, Z. C. Effect of camellia oleifera essential oil on transdermal delivery of aconitine from carbopol gel. Int. J. Integr. Biol. 2009, 1, 58-62.

[17] Hu, X. F.; Liu, S. X.; Bi, J. Y. Research progress of transdermal absorption of ligustrazine. J. Liaoning Univ. Tradit. Chin. Med. 2014, 8, 162-164 (in Chinese).

[18] Wei, S.; Zhi, Q. L.; Shu, M. W. Study on plastics transdermal absorption of berberine hydrochloride of complex prescription coptis chinensis. Lishizhen Med. Mater. Med. Res. 2009, 20, 2696-2697.

[19] Liu, R.; Chu, X.; Sun, A. Preparative isolation and purification of alkaloids from the Chinese medicinal herb evodia rutaecarpa (Juss.) Benth by high-speed counter-current chromatography. J. Chromatogr. A 2005, 1074, 139-144.

[20] Zhao, Q.; Zhang, R. T.; Zhang, Jun. Investigation on in vitro transdermal absorption of evodia rhizoma powder and its main active ingredients. Chin. Med. J. 2019, 34, 1730-1734 (in Chinese).

[21] Lai, J. P.; He, X. W.; Jiang, Y. Preparative separation and determination of matrine from the Chinese medicinal plant sophora flavescensait by molecularly imprinted solid-phase extraction. Anal. Bioanal. Chem. 2003, 375, 264-269.

[22] Wei, H.; Fu, Z.; Mu, J. Investigation of microemulsion system for transdermal delivery of matrine. Adv. Mat. Res. 2011, 284, 219-225.

[23] Shang, K.; Li, J. W.; Chang, M. Y. Study on chemical composition and pharmacological effect of borneol. Jilin J. Tradit. Chin. Med. 2018, 38, 93-95 (in Chinese).

[24] Chen, M.; Yu, Y. I. Research progress on chemical composition and biological activity of eucalyptus oil. Chin. J. Mod. Med. 2014, 4, 97-100 (in Chinese).

[25] Han, J. Research progress on the chemical composition of peppermint and its role in promoting transdermal absorption. China School Doctor 2006, 20, 109-110 (in Chinese).

[26] Cristina, P.; Silvia, P.; Sara, N. New insights on the mechanism of fatty acids as buccal permeation enhancers. Pharmaceutics 2018, 10, 201.

[27] Pang, L. L.; Xiong, W. Z.; Li, L. Analysis of characteristics research progress of transdermal permeation of TCM chemical components. J. Tradit. Chin. Med. 2019, 4, 737-741 (in Chinese).

[28] Zhang, T. Advances in the study of percutaneous absorption promotion of aromatic Chinese medicinal components. Chin. J. Tradit. Med. 2019, 34, 2589-2592 (in Chinese).

[29] Zhang, Y. H.; Zeng, H. P. Research progress on the role of fatty 
acids in life. China Oils Fats 2006, 31, 11-16 (in Chinese).

[30] Mittal, A.; Sara, U.; Ali, A. Status of fatty acids as skin penetration enhancers a review. Curr. Drug Delivery 2009, 6, 274-279.

[31] Tao, D. Q. Research progress of supercritical $\mathrm{CO}_{2}$ extraction of medicinal oils and fatty acids in natural medicine. Anhui Med. 2011, 8, 114-116 (in Chinese).

[32] Dimas, D. A.; Dallas, P. P.; Rekkas, D. M. Ion pair formation as a possible mechanism for the enhancement effect of lauric acid on the transdermal permeation of ondansetron. Pharm. Dev. Technol. 2005, 9, 311-320.

[33] Esmail, M. Influence of oleic acid and other permeation promoters on transdermal delivery of dihydroergotamine through rabbit skin. Int. J. Pharm. 1991, 1, 97-100.

[34] Yamane, M. A.; Williams, A. C.; Barry, B. W. Effects of terpenes and oleic acid as skin penetration enhancers towards 5 -fluorouracil as assessed with time; permeation, partitioning and differential scanning calorimetry International. Int. J. Pharm. 1995, 116, 237-251.

[35] Jain, A. K.; Panchagnula, R. Transdermal drug delivery of tricyclic antidepressants: effect of fatty acids. Methods Find. Exp. Clin. Pharmacol. 2003, 25, 413.

[36] Aungst, B. J.; Blake, J. A.; Rogers, N. J. Transdermal oxymorphone formulation development and methods for evaluating flux and lag times for two skin permeation enhancing vehicles. $J$. Pharm. Sci. 1990, 79, 1072-1076.

[37] Aungst, J. Structure/Effect studies of fatty acid isomers as skin penetration enhancers and skin irritants. Pharm. Res. 1989, 6, 244-247.

[38] Xi, G. Q.; Shen, S. G.; Wang, J. S. Study on the effect of oleic acid on the percutaneous penetration of nitrendipine cream. J. Handan Univ. 2005, 3, 63-65 (in Chinese).

[39] Zhou, X.; He, C. X.; Sulitan, A. Research progress of alkaloids. Biotechnology Newsletter 2006, 17, 476-479 (in Chinese).

[40] Renner, U. D.; Oertel, R.; Kirch, W. Pharmacokinetics and pharmacodynamics in clinical use of scopolamine. Ther. Drug Monit. 2005, 27, 655-665.

[41] Wang, X. Research progress of patch of Chinese medicine alkaloid. Chin. Herb. Med. 2011, 42, 1857-1861 (in Chinese).

[42] Yun, L. I.; Shu, C. W.; Yong, Q. L. Influences of different alkaloid penetration enhancers on transdermal absorption of paeoniflorin. $J$. Beijing Univ. Tradit. Chin. Med. 2014, 37, 410-413.

[43] Xu, J. F. Experimental study on percutaneous penetration behavior of 17 alkaloids in vitro. PLA Pharm. J. 2001, 17, 66-68 (in Chinese).

[44] Li, W. H.; Wang, Y. Z.; Luo, S. X. Study on transdermal mechanism of sophora flavescens alkaloids nanoemulsion and nanoemulsion based gels. Chin. Tradit. Herb. Drugs 2017, 48, 484-489.

[45] Jun, C.; Jin, Q. D.; Chen, Y. P. Natural terpenes as penetration enhancers for transdermal drug delivery. Molecules 2016, 21,
1709.

[46] El-Kattan, A. F.; Asbill, C. S.; Kim, N. The effects of terpene enhancers on the permeation of drugs with different lipophilicities. Int. J. Pharm. 2001, 43, 229-240.

[47] Ahad, A.; Aqil, M.; Kohli, K. Role of novel terpenes in transcutaneous permeation of valsartan: effectiveness and mechanism of action. Drug Dev. Ind. Pharm. 2011, 37, 583-596.

[48] Vaddi, H. K.; Ho, P. C.; Chan, S. Y. Terpenes in propylene glycol as skin penetration enhancers: Permeation and partition of haloperidol, fourier transform infrared spectroscopy, and differential scanning calorimetry. J. Pharm. Sci. 2002, 91, 1639-1651.

[49] Aqil, M.; Ahad, A.; Sultana, Y. Status of terpenes as skin penetration enhancers. Drug Discov. Today 2007, 12, 1061-1067.

[50] Shen, G.; Jagdish, S. Mechanism of transdermal transport of 5-fluorouracil by terpenes: carvone, 1,8-cineole and thymol. Int. J. Pharm. 1997, 154, 67-77.

[51] Lim, P. F. C.; Liu, X. Y.; Chan, S. Y. A review on terpenes as skin penetration enhancers in transdermal drug delivery. J. Essent. Oil Res. 2009, 21, 423-428.

[52] Wang, J.; Dong, C.; Song, Z. Monocyclic monoterpenes as penetration enhancers of ligustrazine hydrochloride for dermal delivery. Pharm. Dev. Technol. 2017, 22, 571-577.

[53] Jin, H. H.; Han, H. L.; Zheng, G, H. Study on the mechanism of duxiang terpene promoting transdermal absorption. Med. J. Yanbian Univ. 2001, 24, 25-29 (in Chinese).

[54] Yum, S. I. Transdermal therapeutic systems and rate control drug delivery. Med. Prog. Technol. 1989, 15, 47-52.

[55] Li, Y. Z.; Xie, Y.; He, Y. F. Quality markers of traditional Chinese medicine: concept, progress and perspective. Engineering 2019, 5, 888-894.

[56] Liu, J. H.; Wang, H.; Qu, P. F. Study on transdermal absorption of berberine in Jinhuang salve in vitro. Chin. Tradit. Pat. Med. 2014, 36, 65-69 (in Chinese).

[57] Yang, H. J.; Wang, Z. Y.; Liu, H. Effect of natural borneol on transdermal absorption of compound Chinese medicine eczema cream in vitro. Chin. J. Exp. Tradit. Med. Form. 2017, 28, 2650 (in Chinese).

[58] Pikal, M. J. The role of electroosmosis flow in transdermal iontophoresis. Adv. Drug Deliv. Rev. 2001, 46, 281-305.

[59] Li, W. Z.; Hao, B. H.; Deng, Z. H. Effects of different penetration enhancers on transdermal absorption of sinomenine under electroporation conditions. Chin. Herb. Med. 2006, 37, 1790-1792.

[60] Ita, K. Transdermal delivery of drugs with microneedles: strategies and outcomes. J. Drug Deliv. Sci. Technol. 2015, 29, 16-23.

Received May 21, 2020

Accepted August 13, 2020 